\title{
Selective Enhancement of Processing of Negative Faces During Slow-Wave Sleep
}

\author{
Simon Barton ${ }^{1 *}$, Reinhard Pietrowsky ${ }^{1}$ \\ 1 University of Düsseldorf, Institute for Experimental Psychology, Clinical Psychology
}

\begin{abstract}
The association between napping and consolidation of emotional face memory is still not well understood. In the present study, subjects of a nap and wake condition (each $n=20$ ) were presented pictures of facial expressions in a declarative memory task and word learning was used as a control task, whereby emotional valence in both tasks was varied. Hypothesized was better memory performance for negative than neutral stimuli after a $1 \mathrm{~h}$ nap as well as a positive association between negative stimuli and non-rapid eye movement (NREM) sleep. Memory performance was evaluated via face recognition (old/new discrimination) and free recall of words. No enhancement of face recognition and word recall by sleep was found. However, while recall of negative words positively correlated with NREM sleep (i.e., total sleep time), the positive and linear association for recognition of negative faces was limited to the sleep stage N3 (rich in slow-wave sleep). Selective enhancement of negative facial processing by slow-wave sleep can be concluded.

Keywords: Memory consolidation, face memory, nap, emotional enhancement, NREM sleep, slow-wave sleep.
\end{abstract}

\section{Introduction}

Sleep following learning has a beneficial effect on memory consolidation (Diekelmann, Wilhelm, \& Born, 2009), which will in the present study be referred to as the sleep-memory-effect. Sleep is distinguished between rapid eye movement (REM) sleep and non-REM (NREM) sleep, with the latter comprising the stages $\mathrm{N}_{1}, \mathrm{~N}_{2}$ and N3. The deepest NREM stage $\mathrm{N}_{3}$ is rich of slow-wave sleep (SWS)

*Correspondence: Simon.Barton@hhu.de, University of Düsseldorf, Institute for Experimental Psychology, Clinical Psychology, Universitätsstr. 1, 40225 Düsseldorf, Germany

Received: 26 September 2018 Accepted: 10 August 2019

Sleep and Hypnosis

Journal homepage:

http://www.sleepandhypnosis.org

ISSN:1302-1192 (Print) 2458-9101 (Online)
(Iber, Ancoli-Israel, Chesson, \& Quan, 2007). It is assumed that SWS primarily benefits hippocampusdependent declarative memory consolidation, while REM sleep primarily benefits non-declarative memory consolidation (Plihal \& Born, 1997). A nap of 60 min has been shown to improve declarative memory for word pairs and word recall correlated with NREM sleep (Tucker et al., 2006), and even an ultra-short 6 min nap improves word recall (Lahl, Wispel, Willigens, \& Pietrowsky, 2008).

"Emotional enhancement" refers to the finding that memory consolidation is superior for emotional rather than neutral information (Wagner, Kashyap, Diekelmann, \& Born, 2007), especially information with a negative valence, e.g., negatively arousing words compared to neutral words (Kensinger \& Corkin, 2003). Sleep especially benefits the consolidation of emotionally arousing 
declarative memories, e.g., emotional pictures (Hu, Stylos-Allan, \& Walker, 2006). Research has long argued for an association of this effect with REM sleep (e.g., Diekelmann et al., 2009; Groch, Wilhelm, Diekelmann, \& Born, 2013). After a 90 min nap, memory consolidation for negative, but not for matched neutral pictures was enhanced and positively correlated with the amount of REM sleep (Nishida, Pearsall, Buckner, \& Walker, 2009). However, Cellini, Torre, Stegagno, and Sarlo (2016) demonstrated enhanced memory consolidation for emotional and neutral pictures independent of REM sleep after a 90-120 min nap before and after learning. The authors suggested that NREM sleep promotes emotional memory formation, which was previously argued by Ackermann and Rasch (2014).

Superior memory for faces has been found for negative facial expressions (Johansson, Mecklinger, \& Treese, 2004; Wang, 2013) and positive faces (D'Argenbeau, Van der Linden, Comblain, \& Etienne, 2003; Shimamura, Ross, \& Bennett, 2006). So far, little research regarding the sleep-memory-effect for face recognition has been done. Wagner et al. (2007) investigated memory consolidation for declaratively learned facial expressions (happy, angry, and neutral) after nocturnal sleep. Face recognition after two days was more accurate in the sleep condition, independent of valence. NREM sleep was positively correlated with memory accuracy for emotional faces. In general, negative faces are better recognized than positive and neutral faces (Wang, 2013).

Although there is evidence that NREM sleep plays a critical role in emotional declarative memory and declarative face memory, a beneficial effect of NREM sleep for memory consolidation of emotional faces has not yet been found. However, in previous studies, valence was either not varied (Clemens, Fabó, \& Halász, 2005; Wagner, Hallschmid, Verleger, \& Born, 2002) or emotional faces were presumably not sufficiently arousing (Kashyap, 2014; Wagner et al., 2007). Superior memory for negative over positive content is generally assumed (Kensinger \& Corkin, 2003; Wang, 2013). The purpose of the present study was to explore the isolated effects of NREM sleep during a nap for memory of negative faces in a declarative recognition task with emotional facial expressions. Word learning was additionally tested as a control condition to verify a sleep-memory-effect since a sleep-induced enhancement for word recall is more firmly established (Lahl et al., 2008; Tucker et al., 2008). Hypotheses were the following:

1. Across all subjects, negative stimuli are significantly better remembered than neutral ones (main effect of valence).

2. Following sleep, significantly more stimuli are remembered than following wakefulness (sleep-memory-effect).

3. Following sleep, significantly more negative stimuli are remembered than following wakefulness (emotional sleep-memory-effect).

4. There is a significant linear positive correlation between NREM sleep and memory for negative stimuli.

\section{METHOD}

\section{Subjects}

The sample consisted of 40 subjects, who were randomly assigned to either the sleep condition ( $n=20$ ) or the wake condition $(n=20)$. Subjects were recruited at the university and most were students $(n=34)$. All subjects were either native German speakers or fluent in the German language. Exclusion criteria were sleep disorders, prosopagnosia, mental disorders, intake of drugs and neurological disorders. Age was restricted to $18-50$ years. Subjects received financial compensation or credit points for participation. The sleep group consisted of 12 males and 8 females aged $25.05 \pm 6.88$ years, while the wake group consisted of 6 males and 14 females aged 27.05 \pm 6.82 years. For the day of testing, subjects were instructed to refrain from sleeping past 8:00 AM and from drinking beverages containing caffeine or alcohol later than 6:00 PM on the day before, which they documented in a sleep diary. The study was approved by the local ethics-committee and written informed consent was obtained from all participants. 


\section{MATERIALS and MEASUREMENTS}

\section{Face recognition task}

Pictures depicting especially intensive facial expressions were chosen from the Karolinska Directed Emotional Faces (KDEF) database (Lundqvist, Flykt, \& Öhman, 1998). Each picture could be unambiguously categorized as either having a positive (laughing), negative (angry) or neutral valence. During the learning session, 30 pictures were presented (10 per valence, with each valence containing 5 male and 5 female faces). Each person appearing on the pictures was presented only once. Presentation time and the interstimulus interval (ISI) were both set to $1,000 \mathrm{~ms}$. Order of pictures was counterbalanced between subjects. Different numbers of pictures, presentation times and ISIs were tested in a prestudy $(n=12)$. A presentation time and ISI longer than 1,000 $\mathrm{ms}$ as well as less than 30 learning pictures made the task too undemanding; therefore, a shorter presentation time was used than by Wagner et al. (2007). The recognition task (after the retention interval) consisted of 60 pictures: the previously shown 30 pictures (identical valence expressions) and 30 novel pictures (also 10 per valence, with each valence containing 5 male and 5 female). Pictures were presented consecutively without time limitation. Subjects had to choose if the presented picture was familiar or new. Order of pictures in both the learning session and recognition task was randomized. Visual recognition can be tested only once for the same material in order to prevent relearning (Clemens et al., 2005; Wagner et al., 2007).

\section{Word recall task}

Subjects were given a list of 30 German nouns of positive (e.g., love, smile), negative (e.g., murder, tumor) and neutral (e.g., building, event) valence with 10 from each valence category, generated from previous studies using emotional words (e.g., Richards, French, Johnson, Naparstek, \& Williams, 1992). Words between valence categories were matched in length (1-3 syllables) and concreteness. Order of words was counterbalanced between subjects. Memory performance was tested via free recall where subjects had to write down the memorized words. The recall task was conducted immediately after learning to determine memory baseline and after the retention interval. Learning time as well as number and difficulty of words were tested before in a prestudy $(n=12)$, where a learning time longer than 2 min and very short words (1 syllable) resulted in a too low difficulty level.

\section{Computer and EEG material}

The face recognition task was presented on a 28 " monitor with a $1440 \times 900$ resolution. The operating system was Windows 7 and the picture task was programmed and presented with Inquisit 3. During the preparation, the skin on the head of each subject was cleaned with an abrasive cleaning emulsion and isopropanol (70 \%). Ten $\mathrm{Ag} / \mathrm{AgCl}$ chloride electrodes filled with conductive paste were used for EEG measurement. Sleep-EEG was measured via the portable device SOMNOscreen and evaluated with DOMINO 2.6.0 software.

\section{Design}

The between-subjects variable group (nap and wake during the retention interval) and withinsubjects variable valence (positive, negative, and neutral) were varied across pictures and words. Additionally, the word recall task included the within-subjects variable recall (after learning and after the retention interval). For the face recognition task, a 2 (group) x 3 (valence) between-subjects design was implemented (as a within-subjects design would produce familiarity with the stimulus material, therefore possibly obscuring an effect of sleep), while the word recall task consisted of a 2 (group) $\times 2$ (recall) x 3 (valence) between-within-subjects design. Dependent variables were the average number of recognized faces and recalled words.

\section{Procedure}

The experiment started either at midday or in the early afternoon. Testing took place in an electrical and sound attenuated $2.44 \times 3.46 \mathrm{~m}$ booth. After giving general information about the experiment, clarifying the exclusion criteria and receiving written consent, the subjects were randomly assigned to either the sleep or wake condition. Subjects then gave sociodemographic information. For the sleep group, EEG electrodes were placed according to the international 10/20 
system. Head electrodes were placed at $\mathrm{C}_{3}, \mathrm{Cz}$ and $\mathrm{C}_{4}$, reference electrodes on the mastoids, the ground electrode on the forehead, and the EMG electrode on the chin. EOG electrodes were used for measurement of vertical (VEOG, lateral of the left eye) and horizontal (HEOG, above and below the right eye) eye movements. Impedance was kept below $5 \mathrm{kOhm}$.

For the learning session, subjects were placed in front of the computer with a distance of $68 \mathrm{~cm}$ between the face and monitor. Subjects received intentional learning instructions, i.e., they were informed that memory tests would follow. First, subjects were presented the faces in a learning session and instructed to memorize each picture. Second, they were given the word list with $2 \mathrm{~min}$ time to memorize as many words as possible regardless of the word order. Afterwards, subjects were instructed to write down all the memorized words. Recall time was limited to $10 \mathrm{~min}$.

The subsequent $1 \mathrm{~h}$ retention interval always took place between 1:00 - 4:00 PM to control for circadian effects. Subjects of the sleep group laid on a bed provided to them in the booth. Sleep was recorded by polysomnography and monitored online throughout the retention interval. Subjects of the wake group watched television episodes depicting animal puppets (i.e., no human faces) and were constantly monitored to stay awake. After $1 \mathrm{~h}$ of time in bed, subjects of the sleep group were awakened. Subjects of the sleep group waited 5 min until being fully awake. During this time, the electrodes were removed, while subjects of the wake group waited the same amount of time for comparison.

For the face recognition task, subjects were instructed to decide for each picture whether it was "old" (previously seen in the learning session) or "new" (seen for the first time). Subjects used a computer mouse to choose one of these options. Immediately afterwards, a second free recall of words took place with 2 minutes time to write the recalled words down as before. Finally subjects received compensation for participation.

\section{Data Analysis}

Statistical analysis was conducted with SPSS Statistics 24. All analyses were two-sided and had a significance level of $p \leq .05$. Variances are indicated with standard error (SE). Effect sizes are indicated with partial Eta-squared (for analysis of variance, ANOVA) and Cohen's d (for t-test).

For the face recognition task, recognition memory was calculated as memory accuracy $[\mathrm{Pr}=\mathrm{HR}-\mathrm{FAR}]$ by subtracting the false alarm rate $[F A R=$ proportion of new faces falsely categorized as "old"] from the hit rate $[\mathrm{HR}=$ proportion of old faces correctly categorized as "old"]. In addition, the response bias $[\mathrm{Br}=\mathrm{FAR}$ / ( 1 - Pr)] was computed, i.e., the general tendency to classify stimuli as familiar despite uncertainty (Snodgrass \& Corwin, 1988; Wagner et al., 2007). An ANOVA was conducted including the between-subjects variable group (nap and wake) and the within-subjects variable valence (positive, negative, and neutral). Significant effects were specified by follow-up t-tests. The dependent variables were Pr per valence category.

For exploratory reasons, the reaction time (RT) during face recognition was analyzed. For RT, an ANOVA included the between-subjects variable group (nap and wake) as well as the within-subjects variables old/new (old faces and new faces) and valence (positive, negative, and neutral).

For the word recall task, a repeated measures ANOVA was calculated including the-between-subjects variable group (nap and wake) as well as the withinsubjects variables recall (after learning and after the retention interval) and valence (positive, negative, and neutral). Follow-up t-tests were applied to specify significant effects. The dependent variables were the averaged number of recalled words per valence category.

Sleep parameters were scored manually according to standard criteria (Iber et al., 2007). Pearson correlation coefficients (two-tailed) were calculated for the relation of sleep parameters (time in min as applied by Lahl et al., 2008) with face recognition as well as word recall, respectively. Regarding word recall, the difference in memory performance between both recall tasks was computed, i.e., recall after learning subtracted from recall after the retention interval for each valence category. A negative prefix indicates a decline in memory performance with fewer reproduced words in the second recall, while a positive prefix indicates an increase in memory performance. 


\section{RESULTS}

\section{Sleep Parameters}

The sleep EEG showed that all 20 subjects of the sleep group were able to fall asleep. Sleep parameters are presented in Table 1. Total sleep time (TST) was on average $34.84 \pm 3.69 \mathrm{~min}$, thus slightly more than half the time spent in bed. Subjects entered the N1 sleep stage on average after $10.26 \pm 1.99 \mathrm{~min}$. Sleep was dominated by $\mathrm{N}_{2}(51.69 \pm 7.15 \%)$ and $\mathrm{N}_{3}$ sleep (30.74 $\pm 8.07 \%$ ). Twelve subjects entered $\mathrm{N}_{3}$ sleep (i.e., SWS $>0$ ). These subjects with $\mathrm{N}_{3}>0$ had a higher TST with an average of $46.78 \pm 1.39 \mathrm{~min}$ and entered N1 sleep earlier on average after $5.83 \pm 0.89$ min. For this subsample, sleep was also dominated by $\mathrm{N} 2(50.32 \pm 7.39 \%)$ and $\mathrm{N}_{3}$ sleep $(38.14 \pm 9.60$ $\%)$. None of the subjects entered REM sleep. Eight subjects of the sleep group admitted to be habitual midday sleepers, though only infrequently. Time in bed during night before the experiment was $7.55 \pm$ $0.23 \mathrm{~h}$ for the sleep group and $7.29 \pm 0.55 \mathrm{~h}$ for the wake group, which did not significantly differ $(t(25)$ $=0.43, p=.673, d=0.02$ ). All subjects indicated to be awake before 8:00 AM.

\section{Analysis of the Face Recognition Task}

The main effect of valence for Pr across all subjects was significant $\left(F(2,37)=4.41, p=.019, \eta^{2}=0.19\right)$ with positive faces $(0.50 \pm 0.03)$ being significantly more accurately recognized than neutral faces $(0.40 \pm 0.04 ; t(39)=2.90, p=.006, d=0.46)$ and negative faces $(0.40 \pm 0.04 ; t(39)=2.50, p=.017, d$
$=0.40)$, while recognition between the neutral and negative valences did not differ $(t(39)=0.00, p=$ 1.00o). Therefore, the first hypothesis that negative faces will be better recognized than neutral faces is rejected.

The results of the face recognition task for both groups are shown in Table 2. For Pr, there was neither a significant main effect of group $(F(1,38)$ $=0.00, p=1.000$ ) nor an interaction with valence $(F(2,37)=0.48, p=.626)$. Thus, the second hypothesis of generally better recognition for faces after sleep is rejected. Likewise, the third hypothesis of better recognition specifically of negative faces after sleep is rejected.

For $\mathrm{Br}$, there were no significant effects for valence $(F(2,37)=1.49, p=.240)$, group $(F(1,38)=$ $0.00, p=.999)$ as well as no significant interaction $(F(1,37)=0.61, p=.549)$. No significant effects for RT were found.

\section{Analysis of the Word Recall Task}

For the recall difference, a significant main effect of valence across all subjects was found $(F(2,37)=$ 10.81, $\left.p<.001, \eta^{2}=0.37\right)$. Compared to neutral words $(3.84 \pm 0.27)$, positive $(4.94 \pm 0.23 ; t(39)=$ $4.16, p<.001, d=0.66)$ and negative words (4.98 $\pm 0.23 ; t(39)=4.08, p<.001, d=0.64)$ were significantly better recalled, while recall of positive and negative valence words did not significantly differ $(t(39)=-0.14, p=.889)$. Therefore, the results confirm the first hypothesis of better remembered negative words than neutral words.

Table 1: Sleep parameters for subjects of the sleep group.

\begin{tabular}{|c|c|c|c|c|}
\hline & \multicolumn{2}{|c|}{$\begin{array}{l}\text { All subjects of the } \\
\text { sleep group }(n=20)\end{array}$} & \multicolumn{2}{|c|}{$\begin{array}{l}\text { Subjects with } \\
\text { N3 > } 0(n=12)\end{array}$} \\
\hline & $\mathbf{M}$ & SE & M & SE \\
\hline TST & 34.84 & 3.69 & 46.78 & 1.39 \\
\hline N1-SOL & 10.26 & 1.99 & 5.83 & 0.89 \\
\hline $\mathrm{N} 1$ in $\min$ & 6.12 & 1.25 & 5.40 & 1.87 \\
\hline $\mathrm{N} 1$ in $\%$ & 17.57 & 3.58 & 11.54 & 5.36 \\
\hline $\mathrm{N} 2$ in $\min$ & 18.01 & 2.49 & 23.54 & 2.57 \\
\hline N2 in \% & 51.69 & 7.15 & 50.32 & 7.39 \\
\hline N3 in min & 10.71 & 2.81 & 17.84 & 3.34 \\
\hline N3 in \% & 30.74 & 8.07 & 38.14 & 9.60 \\
\hline
\end{tabular}

Note. TST: Total sleep time; N1-SOL: stage 1 sleep onset latency; N1: stage 1 sleep; N2: stage 2 sleep; N3. stage 3 sleep, N3 > o: subjects who entered N3 sleep, min: minutes. 
Table 2: Recognized faces of sleep and wake groups.

\begin{tabular}{|c|c|c|c|c|}
\hline & \multicolumn{2}{|c|}{$\begin{array}{l}\text { Sleep group } \\
\qquad(\mathrm{n}=\mathbf{2 0})\end{array}$} & \multicolumn{2}{|c|}{$\begin{array}{l}\text { Wake group } \\
\qquad(n=20)\end{array}$} \\
\hline & M & SE & M & SE \\
\hline \multicolumn{5}{|l|}{ Hit Rate (HR) } \\
\hline Positive faces & 0.70 & 0.04 & 0.66 & 0.04 \\
\hline Negative faces & 0.65 & 0.04 & 0.66 & 0.05 \\
\hline Neutral faces & 0.61 & 0.05 & 0.63 & 0.05 \\
\hline All faces & 0.65 & 0.04 & 0.65 & 0.03 \\
\hline \multicolumn{5}{|c|}{ False alarm rate (FAR) } \\
\hline Positive faces & 0.20 & 0.04 & 0.17 & 0.03 \\
\hline Negative faces & 0.25 & 0.04 & 0.27 & 0.04 \\
\hline Neutral faces & 0.23 & 0.05 & 0.22 & 0.03 \\
\hline All faces & 0.22 & 0.03 & 0.22 & 0.03 \\
\hline \multicolumn{5}{|c|}{ Memory accuracy (Pr) } \\
\hline Positive faces & 0.51 & 0.05 & 0.49 & 0.04 \\
\hline Negative faces & 0.41 & 0.05 & 0.39 & 0.05 \\
\hline Neutral faces & 0.38 & 0.05 & 0.42 & 0.06 \\
\hline All faces & 0.43 & 0.04 & 0.43 & 0.04 \\
\hline \multicolumn{5}{|c|}{ Response Bias (Br) } \\
\hline Positive faces & 0.40 & 0.08 & 0.33 & 0.05 \\
\hline Negative faces & 0.40 & 0.06 & 0.43 & 0.05 \\
\hline Neutral faces & 0.31 & 0.06 & 0.36 & 0.05 \\
\hline All faces & 0.38 & 0.05 & 0.38 & 0.04 \\
\hline
\end{tabular}

Note. HR: proportion of old faces correctly categorized as "old"; FAR: proportion of new faces falsely categorized as "old", Pr: memory accuracy [Pr = HR - FAR], Br: response bias [Br = FAR / (1 - Pr)]

Memory performance in both recall conditions as well as the difference in recall for each group is shown in Table 3. There was a significant main effect of recall across all subjects $(F(1,38)=65.26$, $\left.p<.001, \eta^{2}=0.63\right)$ with on average more words remembered at recall after learning (4.99 \pm 0.19$)$ than after the retention interval $(4.18 \pm 0.20)$. Recall did not significantly interact with valence $(F(2,37)=0.98, p=.386)$. The factor group did not exert an influence on memory performance $(F(1,38)=0.28, p=.598)$, and did not interact with recall $(F(1,38)=0.03, p=.870)$, valence $(F(2,37)=$ $0.37, p=.695)$ or both of these variables $(F(2,37)$ $=1.34, p=.273)$. Thus, the second hypothesis of generally better memory for words after sleep is rejected. Likewise, the third hypothesis of better memory specifically for negative words after sleep is rejected. 
Table 3: Recalled words of sleep and wake group

\begin{tabular}{|c|c|c|c|c|}
\hline & \multicolumn{2}{|c|}{$\begin{array}{l}\text { Sleep group } \\
\qquad(n=20)\end{array}$} & \multicolumn{2}{|c|}{$\begin{array}{l}\text { Wake group } \\
\qquad(n=20)\end{array}$} \\
\hline & M & SE & M & SE \\
\hline \multicolumn{5}{|l|}{ R1 } \\
\hline Positive words & 5.20 & 0.38 & 5.45 & 0.32 \\
\hline Negative words & 5.45 & 0.34 & 5.50 & 0.28 \\
\hline Neutral words & 4.00 & 0.40 & 4.35 & 0.41 \\
\hline Sum of words & 14.65 & 0.93 & 15.30 & 0.68 \\
\hline \multicolumn{5}{|l|}{$\mathbf{R 2}$} \\
\hline Positive words & 4.60 & 0.31 & 4.50 & 0.36 \\
\hline Negative words & 4.45 & 0.40 & 4.50 & 0.36 \\
\hline Neutral words & 3.20 & 0.41 & 3.80 & 0.39 \\
\hline Sum of words & 12.25 & 0.87 & 12.80 & 0.81 \\
\hline \multicolumn{5}{|l|}{ R2-R1 } \\
\hline Positive words & -0.60 & 0.18 & -0.95 & 0.19 \\
\hline Negative words & -1.00 & 0.30 & -1.00 & 0.23 \\
\hline Neutral words & -0.80 & 0.17 & -0.55 & 0.26 \\
\hline Sum of words & -2.40 & 0.43 & -2.50 & 0.43 \\
\hline
\end{tabular}

Note. $\mathrm{R} 1$ = recall after learning, $\mathrm{R} 2$ = recall after retention, R2-R1: recall difference (a negative prefix means a decline in word recall from $\mathrm{R} 1$ to $\mathrm{R} 2$ ).

\section{Correlations between Memory Performance and Sleep Parameters}

Correlations between memory performance and sleep parameters are shown in Table 4. For face recognition, Pr for negative faces significantly and positively correlated with $\mathrm{N}_{3}$ sleep $(r=.598, p=$ .005). This positive correlation between N3 and recognition of negative faces was significantly higher for the N3 $>$ o subsample $(r=.645, p=.024)$, as shown in Fig. 1. Furthermore, there were significant negative correlations between sleep onset latency and Pr for negative faces $(r=-.536, p=.015)$ as well as Pr for all faces $(r=-.492, p=.028)$, i.e., early sleep onset led to an increase in memory accuracy for faces, especially negative faces. For word recall, there was a significant positive correlation between recall difference for negative words with TST ( $r$ $=.555, p=.011)$ and N3 sleep $(r=.510, p=.022)$. The positive correlation between $\mathrm{N}_{3}$ and recall of negative words did not reach significance for the $\mathrm{N}_{3}$ $>$ o subsample $(r=.331, p=.293)$. There was also a negative correlation between sleep onset latency and recall difference for negative words $(r=-.565$, $p=$.009), i.e., early sleep onset led to an increase in negative words being recalled. Therefore, the hypothesis of a linear positive correlation of NREM sleep with both recognition of negative faces and recall of negative words is confirmed. However, this linear positive correlation of recognition of negative faces was shown only for N3 sleep. 
Table 4: Correlations (two-tailed) between memory performance and sleep parameters (in min).

\begin{tabular}{|c|c|c|c|c|c|c|}
\hline & TST & N1-SOL & N1 & N2 & N3 & N3 $>0$ \\
\hline \multicolumn{7}{|l|}{$\operatorname{Pr}$ (faces) } \\
\hline Positive & $r=.018$ & $r=-.378$ & $r=-.021$ & $r=-.068$ & $r=.092$ & $r=.010$ \\
\hline Negative & $r=.416$ & $r=-.536^{*}$ & $r=-.326$ & $r=.104$ & $r=.598^{* *}$ & $r=.645^{\star}$ \\
\hline Neutral & $r=.275$ & $r=-.376$ & $r=-.228$ & $r=.083$ & $r=.387$ & $r=.255$ \\
\hline Total & $r=.279$ & $r=-.492^{*}$ & $r=-.226$ & $r=.050$ & $r=.422$ & $r=.361$ \\
\hline \multicolumn{7}{|c|}{ R2-R1 (words) } \\
\hline Positive & $r=-.100$ & $r=-.171$ & $r=.149$ & $r=-.300$ & $r=.068$ & $r=.412$ \\
\hline Negative & $r=.557^{\star}$ & $r=-.565^{\star \star}$ & $r=.004$ & $r=.248$ & $r=.510^{*}$ & $r=.331$ \\
\hline Neutral & $r=-.184$ & $r=.187$ & $r=.055$ & $r=-.425$ & $r=.110$ & $r=.530$ \\
\hline Sum & $r=.270$ & $r=-.390$ & $r=.088$ & $r=-.125$ & $r=.426$ & $r=.567$ \\
\hline
\end{tabular}

Note. Pr: memory accuracy; R2-R1: recall difference; TST: total sleep time; N1-SOL: stage 1 sleep onset latency; N1: stage 1 sleep; N2: stage 2 sleep; N3: stage 3 sleep, N3 > 0: subjects who entered N3 sleep. ${ }^{*} p<.05,{ }^{* *} p<.01$.

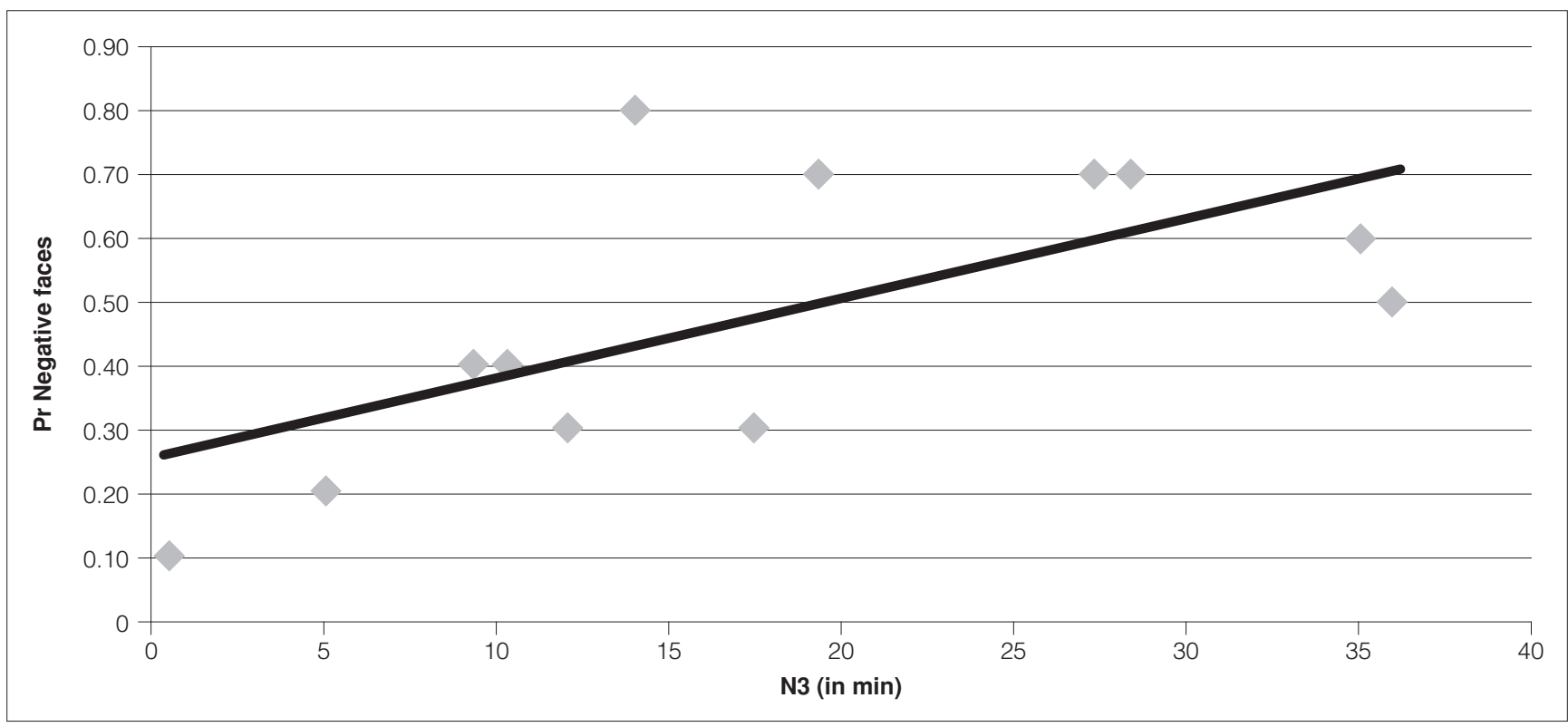

Fig. 1: The linear positive correlation $(r=.645, p=0.24)$ between N3 sleep and memory accuracy (Pr) for negative faces depicted for subjects of the sleep group with $\mathrm{N}_{3}>\mathrm{o}(n=12)$.

\section{DISCUSSION}

Unexpectedly, positive faces were significantly better recognized than negative and neutral faces, while negative and positive words both were significantly better recalled than neutral words. The sleep group did not significantly differ in face recognition and word recall from the wake group, irrespective of valence as well as when specifically investigating negative valence. Memory accuracy for negative faces significantly and positively correlated only with N3 sleep, while the recall of negative words significantly and positively correlated with TST (NREM sleep). A significant positive correlation between recall of negative words and N3 sleep was observed for all subjects of the sleep group, but not for the N3 > o subsample. 
The results of a more accurate recognition of negative faces correlating exclusively with N3 sleep refute Wagner et al. (2007) in that the contribution of SWS to declarative learning would not apply to memory for faces and passive interference reduction as a possible explanation for improved face recognition after sleep. A positive correlation between NREM sleep and memory accuracy for faces was found by Wagner et al. (2007), though also for TST (including REM sleep) and not for specific sleep stages. Tucker et al. (2006) and Payne et al. (2015) argue that a positive correlation between memory improvement and a specific sleep stage, but not TST, demonstrates active memory consolidation. The present study supports this assumption by evidence of a positive correlation between N3 sleep and negative face recognition.

The isolated effects of $\mathrm{N}_{3}$ sleep show a significant positive association with negative faces. This association does not stand in contradiction to the absence of a sleep-memory-effect as memory enhancement by sleep would be the result of TST, hence the length of N3 sleep. Although Lahl et al. (2008) found an effect of sleep for words after shorter TST and $\mathrm{N}_{3}$ sleep, there were differences in methodology and the authors point to a positive association between memory consolidation and TST. The length of the nap and N3 sleep in the present study was not sufficient to enhance face recognition and word recall, but shows selective and more elaborate processing of negative faces. As the association between amount of N3 sleep and negative face memory is correlative, causal conclusions cannot be drawn.

There is evidence in previous research for a positive association between emotional memory and SWS (Ackermann \& Rasch, 2014). SWS selectively consolidated negative information (Payne et al, 2015) and positively correlated with the behavioral consolidation of negative images (Cairney, Durrant, Power, \& Lewis, 2015). While methodologically not directly comparable because of differences in stimulus material and TST, the effects point to the direction of SWS enhancing emotional memory. A possible influence of REM sleep for declarative face memory can be excluded, as none of the subjects in the present study entered this sleep stage.
The correlation between negative face recognition and N3 sleep points to more elaborate processing, and possibly better long-term storage for negative faces. SWS selectively consolidates highly salient stimuli, which are most adaptive to remember (Payne et al., 2015). Aversive stimuli tend to be the most salient (Cairney et al., 2015) and especially recognition of negative faces has an evolutionarily adaptive value (Wang, 2013). There is a general negativity bias in affective processing (Ito, Larsen, Smith, \& Cacioppo, 1998), e.g., faster detection of a negative face in a crowd of happy faces than vice versa (LoBue, 2009).

A beneficial effect of sleep on declarative memory is measured less consistently with recognition than recall (Diekelmann et al., 2009), which could explain the lack of a sleep-memory-effect for face memory. However, also no sleep benefit was found for word recall. Sleep sometimes only has a "long range effect" (after $72 \mathrm{~h}$ ) as consolidation after post-learning sleep continues until memory traces stabilize (Lahl \& Pietrowsky, 2007). In addition, the effect of emotion on memory is time-dependent as it increases with the delay between encoding and testing ( $\mathrm{Hu}$ et al., 2006). It is plausible that memory consolidation in the present study was not completed at retrieval time, thus memory traces may have still been fragile.

Regarding the result that positive faces were most accurately recognized, D'Argembeau et al. (2003) likewise found better memory for intentionally learned positive faces in old/new face discrimination. The authors argue that emotional faces convey self-relevant social feedback and are preferentially processed when positive (e.g., social approval). Although research mostly shows best memory for negative words and pictures, mostly stimulus material was used that does not convey social feedback. Shimamura et al. (2006) attribute better memory for incidentally learned positive expressions to source monitoring, i.e., smiling directs attention to the person (source), while angry faces direct attention to the context (cause of anger). Contradictory results of superior memory for negative faces can be furthermore attributed to methodological differences, such as negative valence category, e.g., fearful faces were shown to 
be remembered best (Wang, 2013). No difference in RT to faces was expected, as none of the subjects entered REM sleep, which is associated with faster responses (Wagner et al., 2007).

Of particular interest is the correlation between the amount of $\mathrm{N}_{3}$ sleep and memory for negative faces. This may cause some speculations about the possible underlying mechanism that accounts for a memory enhancement of negative faces by SWS, which is dominant in the N3 sleep stage. A large number of studies suggest that during SWS, a replay of hippocampal memory traces constitutes the transfer of new memories into long-term memory (e.g., Born, Rasch, \& Gais, 2006; Diekelmann \& Born, 2010; Plihal \& Born, 1997; Tononi \& Cirelli, 2006; Walker, 2009). From these studies, it is suggested that a downscaling of synaptic activity plays an important role in memory consolidation and that this downscaling - likely originating from hippocampal long-term depression - may be one neurophysiological source of the memory enhancing effect of SWS. These previous studies suggest an improved signal-to-noise ratio as a consequence of the removal of redundant neural connections by synaptic downscaling. As suggested by the present results, this function is also modulated by the emotional valence of the to be stored stimuli: negative stimuli (faces) may induce a stronger downscaling and thus a better replay and consolidation, probably due to their adaptive function as threat signals.

Although the present results are small and rather tentative with respect to a better recognition of positive than negative faces, the correlation

\section{References}

Ackermann, S., \& Rasch, B. (2014). Differential effects of non-REM and REM sleep on memory consolidation? Current Neurology and Neuroscience Reports, 14, 1-10.

Born, J., Rasch, B., \& Gais, S. (2006). Sleep to remember. Neuroscientist,12, 410-424.

Cairney, S.A., Durrant, S.J., Power, R., \& Lewis, P.A. (2015). Complementary roles of slow-wave sleep and rapid eye movement sleep in emotional memory consolidation. Cerebral Cortex, 25, 1565-1575.

Cellini, N., Torre, J., Stegagno, L., \& Sarlo, M. (2016). Sleep before and after learning promotes the consolidation of both neutral and emotional information regardless of REM presence. Neuro- between N3 sleep and recall of negative faces seems statistically sound. Nonetheless, future studies should substantiate these results, for example by a second testing after approx. 72-h to clarify a potential long range effect of sleep on memory consolidation (Lahl \& Pietrowsky, 2007), especially for more elaborately processed negative faces. Furthermore, it should be specified whether face recognition after sleep depends on recollection ("remembering") or familiarity-based source memory ("knowing") (Johansson et al., 2004). While enhanced amygdala activity during sleep has primarily been associated with REM sleep (Diekelmann et al., 2009), amygdala activation during SWS after encoding of negative faces should be investigated. Amygdala activation may be a candidate for the emotional modulation of memory consolidation during SWS, since during the learning of emotional stimuli, enhanced amygdala activity and its reactivation during $\mathrm{N}_{3}$ sleep-replay can be assumed.

\section{CONCLUSION}

The present study showed better memory recognition for positive faces and recall for emotional (i.e., positive and negative) words independent of sleep. Recognition for negative faces was positively and linearly correlated with N3 sleep, which points to a selective and more elaborate processing of negative faces during this sleep stage, which is rich in SWS.

The authors do not declare any conflict of interest.

The authors declare that the current study was not financially supported by any commercial institution or organization.

biology of Learning and Memory, 133, 136-144.

Clemens, Z., Fabó, D., \& Halász, P. (2005). Overnight verbal memory retention correlates with the number of sleep spindles. Neuroscience, 132, 529-535.

D’Argenbeau, A., Van der Linden, M., Comblain, C., \& Etienne, A.M. (2003). The effects of happy and angry expressions on identity and expression memory for unfamiliar faces. Cognition \& Emotion, 17, 609-622.

Diekelmann, S., Wilhelm, I., \& Born, J. (2009). The whats and whens of sleep-dependent memory consolidation. Sleep Medicine Reviews, 13, 309-321. 
Diekelmann, S., \& Born, J. (2010). The memory function of sleep. Nature Reviews Neuroscience, 11, 114-126.

Groch, S., Wilhelm, I., Diekelmann, S., \& Born, J. (2013). The role of REM sleep in the processing of emotional memories: Evidence from behavior and event-related potentials. Neurobiology of Learning and Memory, 99, 1-9.

Hu, P., Stylos-Allan, M., \& Walker M.P. (2006). Sleep facilitates consolidation of emotional declarative memory. Psychological Science, 17, 891-898.

Iber, C., Ancoli-Israel, S., Chesson, A., \& Quan, S.F. (2007). The AASM manual for the scoring of sleep and associated events: Rules, terminology and technical specification. American Academy of Sleep Medicine, Westchester.

Ito, T. A., Larsen, J. T., Smith, N. K., \& Cacioppo, J. T. (1998). Negative information weighs more heavily on the brain: The negativity bias in evaluative categorizations. Journal of Personality and Social Psychology, 75, 887-900.

Johansson, M., Mecklinger, A., \& Treese, A.-C. (2004). Recognition of memory for emotional and neutral faces: An event-related potential study. Journal of Cognitive Neuroscience, 16, 1840-1853.

Kashyap, N. (2014). Is emotion perception relative? Evaluating sleep effects on relativity of emotion perception. Psychological Studies, 59, 284-288.

Kensinger, E.A., \& Corkin, S. (2003). Memory enhancement for emotional words: Are emotional words more vividly remembered than neutral words? Memory \& Cognition, 31, 1169-1180.

Lahl, O., \& Pietrowsky, R. (2007). The long range effect of sleep on episodic memory. Sleep and Hypnosis, 9, 24-29.

Lahl, O., Wispel, C., Willigens, B., \& Pietrowsky, R. (2008). An ultra short episode of sleep is sufficient to promote declarative memory performance. Journal of Sleep Research, 17, 3-10.

LoBue, V. (2009). More than just another face in the crowd: superior detection of threatening facial expressions in children and adults. Developmental Science, 12, 305-313.

Lundqvist, D., Flykt, A., \& Öhman, A. (1998). The Karolinska Directed Emotional Faces - KDEF [CD-ROM]. Department of Clinical Neuroscience, Psychology section, Karolinska Institutet, Stockholm.
Nishida, M., Pearsall, J., Buckner, R.L., \& Walker, M.P (2009). REM sleep, prefrontal theta, and the consolidation of human emotional memory. Cerebral Cortex, 19, 1158-1166.

Payne, J.D., Kensinger, E.A., Wamsley, E.J., Spreng, R.N., Alger, S.E., Gibler, K., ..., \& Stickgold, R. (2015). Napping and the selective consolidation of negative aspects of scenes. Emotion, 15, 176-186.

Plihal, W., \& Born, J. (1997). Effects of early and late nocturnal sleep on declarative and procedural memory. Journal of Cognitive Neuroscience, 9, 534-547.

Richards, A., French, C.C., Johnson, W., Naparstek, J., \& Williams, J. (1992). Effects of mood manipulation and anxiety on performance of an emotional Stroop task. British Journal of Psychology, 83, 479-491.

Shimamura, A.P., Ross, J.G., \& Bennett, H.D. (2006). Memory for facial expressions: The power of a smile. Psychonomic Bulletin \& Review, 13, 217-222.

Snodgrass, J.G., \& Corwin, J. (1988). Pragmatics of measuring recognition memory: applications to dementia and amnesia. Journal of Experimental Psychology: General, 117, 34-50.

Tononi, G., \& Cirelli, C. (2006). Sleep function and synaptic homeostasis. Sleep Medicine Reviews, 10, 49-62.

Tucker, M.A., Hirota, Y., Wamsley, E.J., Lau, H., Chaklader, A., \& Fishbein, W. (2006). A daytime nap containing solely nonREM sleep enhances declarative but not procedural memory. Neurobiology of Learning and Memory, 86, 241-247.

Wagner, U., Hallschmid, M., Verleger, R., \& Born, J. (2002). Signs of REM sleep dependent enhancement of implicit face memory: A repetition priming study. Biological Psychology, 62, 197-210.

Wagner, U., Kashyap, N., Diekelmann, S., \& Born, J. (2007). The impact of post-learning sleep vs. wakefulness on recognition memory with different facial expressions. Neurobiology of Learning and Memory, 87, 679-687.

Walker, M.P. (2009). The role of sleep in cognition and emotion. Annals of the New York Academy of Science, 1156, 168-197.

Wang, B. (2013). Facial expression influences recognition memory for faces: Robust enhancement effect of fearful expression. Memory, 21, 301-314. 